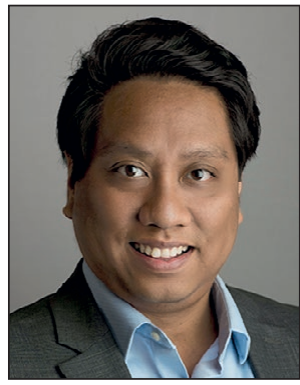

Kristofer G. Reyes is an assistant professor in the Department of Materials Design and Innovation at the University at Buffalo, The State University of New York. He received his PhD degree in applied mathematics from the University of Michigan. He completed postdoctoral research in the Department of Operations Research and Financial Engineering at Princeton University. His research interests include decision-making algorithms and models for autonomous and guided experiments, knowledge elicitation and representation, and high-performance computational materials. Reyes can be reached by email at kreyes3@buffalo.edu.

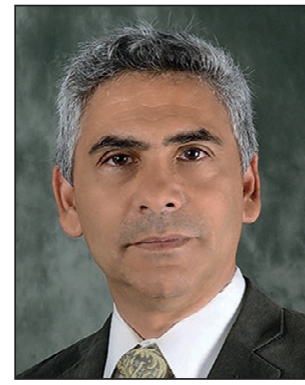

Benji Maruyama is the leader of the Flexible Materials and Processes Research Team, Materials and Manufacturing Directorate, Air Force Research Laboratory. He obtained his PhD degree in materials science and engineering from The University of Texas at Austin. His research interests include autonomous research for materials development and materials synthesis. He developed ARES, the autonomous research robot for materials science. Maruyama can be reached by email at benji.maruyama@us.af.mil.

\title{
International Symposium on Clusters and Nanomaterials to be held November 3-7
}

The 10th International Symposium on Clusters and Nanomaterials (ISCAN) will be held in the historic Jefferson Hotel in Richmond, Va., November 3-7. This symposium is a continuation of the quadrennial Richmond Conference series, which started in 1982. The conference will focus on the structure-property relationships of clusters and nanomaterials, with a focus on their role in solving outstanding problems in energy and medicine-two of the most important challenges facing science and society.

The topics of this symposium include clean and sustainable energy and storage (solar, hydrogen, thermoelectric for energy, and batteries for storage); bioactive, bio-responsive, and biomimetic materials; nanotoxicity; diagnostic and therapeutic devices; bioengineering; and regenerative medicine.

In addition to 14 plenary sessions of invited talks by leading experts and two poster sessions, the symposium will include oral presentations on several hot topics. The symposium will also feature a special memorial session to honor the lives of Millie Dresselhaus, Will Castleman, Walter Kohn, and John Yates, who were associated with this series for many years.

The abstract submission deadline is August 16, and early registration is due August 30 . Additional information can be found at iscan.vcu.edu.

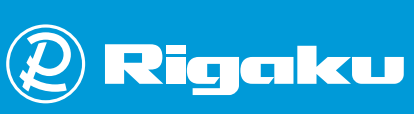

\section{DEEP LEARNING EMPOWERED HIGH-RESOLUTION X-RAY CT}

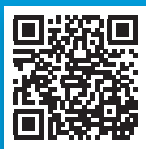

High-resolution X-ray computed tomography (CT) is a great tool to three-dimensionally visualize the complex structure of composite materials, tablets, plants, etc. The Rigaku nano3DX uses low-energy characteristic X-ray radiation $(5.4,8,17 \mathrm{keV})$ to enhance the contrast of light materials. The parallel beam geometry ensures true sub-micron resolution. The high-resolution images allow the analysis of various characteristics such as volume fraction of each component, particle size distribution, fiber orientation, etc. However, the image segmentation - a necessary step before doing any quantitative analysis - can be challenging because a single $X$-ray CT scan often includes thousands of slices of high-resolution images. The ORS Dragonfly software provides an easy-to-use interface to various machine learning and deep learning tools. You can train those programs to segment thousands of images and make challenging and time-consuming image analysis routine.

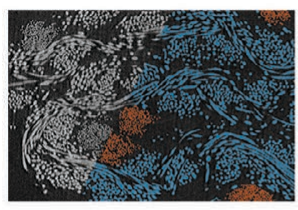

"Tomographic cross section of fiber material segmented using deep learning

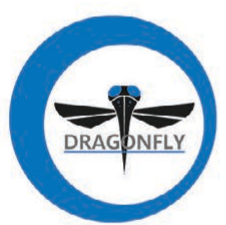

Dragonfly / 3D Visualization and Analysis Software by Object Research Systmes (ORS) Inc.

\section{APP BYTE}

Rigaku Corporation and its Global Subsidiaries www.Rigaku.com | info@Rigaku.com
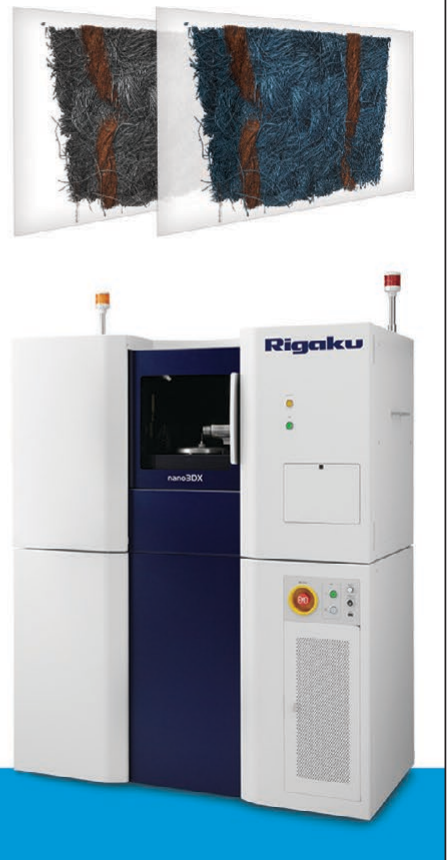